\title{
The latest technology of musculoskeletal ultrasonography: iterative revolution
}

\author{
Masashi Matsuzaki ${ }^{1}$
}

(C) The Japan Society of Ultrasonics in Medicine 2017

\section{Introduction}

The emergence of ultrasound about 60 years ago, at a time when diagnostic imaging was essentially in the form of plain X-rays. The limitation of X-ray for the visualization of gastrointestinal, circulatory, breast/thyroid, gynecological, urinary, and many soft-tissue pathology was far too great. The introduction of ultrasound as a diagnostic tool marked the beginning of the ultimate age of non-radiation investigation. New diagnostic imaging modalities, such as computed tomography (CT) and magnetic resonance imaging (MRI), were subsequently invented, allowing the delineation of multidirectional and high-resolution information on various pathologic conditions. Advances have also been made in the field of ultrasound since those early days. New technologies that utilize the characteristics of ultrasound have been incorporated, allowing it to provide information on various pathologies that other diagnostic imaging modalities cannot provide. Today, ultrasound has achieved the status of being an indispensable diagnostic imaging modality in clinical settings.

\section{Journey of ultrasound in medicine}

Ultrasound currently has five advantages over the other representative diagnostic imaging modalities used in clinical settings, i.e., X-ray, CT, and MRI:

Masashi Matsuzaki

matsuzaki@sonic-j.com

1 Sonic Japan Co., Ltd., Tokyo, Japan
1. Easy to perform tests repeatedly thanks to noninvasive nature.

2. Noninvasive visualization of hemodynamics and tissue properties.

3. Dynamic assessment in real time.

4. Portable devices permits its use outside medical institutions.

5. Cost effectiveness.

Looking back at the journey of abdominal ultrasound, examinations that began with the depiction of organic changes in gallstones and so forth and of space-occupying lesions in the liver, kidneys, and other organs are widely used in clinical settings for screening and similar purposes. Subsequent improvements on image quality have led to advances that allow early detection of masses several millimeters in size, additionally therapeutic procedures that were previously performed under fluoroscopy can now be done under ultrasound guide with improved accuracy and reliability. In the case of liver cancer, treatment used to consist of surgery after diagnostic ultrasound confirmation, but the mainstay of modern treatment consists of minimally invasive procedure, where small tumors measured in millimeters are targeted using ultrasound with pinpoint accuracy. Ultrasound is not only less physically and economically demanding on patients but also greatly contributes to improvement in the quality of medical care.

\section{Journey of musculoskeletal ultrasound}

Looking back at the journey of musculoskeletal ultrasound, $\mathrm{X}$-rays were the first choice for diagnostic imaging of musculoskeletal disorders, since the main target is the skeletal system. Ultrasound was never given a place in this 
area owing to its inability to penetrate bone. Meanwhile, ultrasound of old was given little consideration on softtissue imaging, due to its narrower field of view and poorer spatial resolution at the time as compared with MRI.

However, a direct result of rapid improvements in its close-range spatial resolution in the 21 century, ultrasound has overturned the stereotype that it is not well suited to imaging of bone in the field of musculoskeletal imaging. Not only can it detect bone pathologies that cannot be detected by X-ray, it can now yield information on soft tissue in high resolutions that surpass MRI (Fig. 1). Owing to the current musculoskeletal ultrasound, disorders, such as "pulled elbow" and "ankle sprain", which were so named, because they could not be depicted using imaging modalities in the past, can now be properly visualized and more precisely referred to as "annular ligament dislocation" and "ATFL rupture", respectively. Gaining a better understanding of the precise pathologies led to the formulation of appropriate treatment strategies, confirmation of post-treatment response, and prognostic prediction, resulting in a major evolution from how medicine was practiced in the age of X-rays (Fig. 2). Musculoskeletal ultrasound is also being utilized for on location screening, making maximum use of its noninvasive and portable nature. Growing children, whose skeletal system is fragile, are susceptible to many sports injuries that could be career ending if detection is delayed. Disorders that cannot be depicted using X-rays, such as osteochondritis dissecans and Osgood-Schlatter disease, which are osteochondral sports injuries seen in growing adolescents, can be imaged at the early stage of the disease using musculoskeletal ultrasound (Fig. 3). With musculoskeletal ultrasound screening, serious sequelae can be avoided with early detection and intervention. As such, it has attracted the attention of the sports medicine community.

\section{Latest musculoskeletal ultrasound technology}

Researchers have begun efforts to establish therapeutic procedures for treating, heretofore, the untreatable pain using the latest musculoskeletal ultrasound technology. The keywords here are fascia and fluid. The human trunk is supported by bones, but the trunk is also supported by fasciae which envelop the soft tissue comprising the motor organs along with bone. The bones must move synchronously with the body in order for it to move smoothly. Likewise, soft tissue also supports human movement by synchronizing with this. It has been conjectured that heretofore, the untreatable pain may be caused by an inability of soft tissue enveloped by fasciae to move synchronously with the body due to organic changes and adhesion of the fasciae. In fact, musculoskeletal ultrasound has reached the level, where symptoms can be instantaneously alleviated simply by injecting normal saline into a fascial plane with pinpoint precision at the site of painful symptoms (Fig. 4). There is currently no clear evidence regarding the underlying mechanism of pain relief achieved by the combination of fascial release using fluid. The mechanism by which pain is alleviated may be elucidated in the future by a combination of findings including those based on the basic anatomy and biochemical physiology. The latest musculoskeletal ultrasound technology has begun to evolve tremendously, not only in helping patients who have suffered from chronic pain but also contributing greatly to lowering medical costs in Japan by

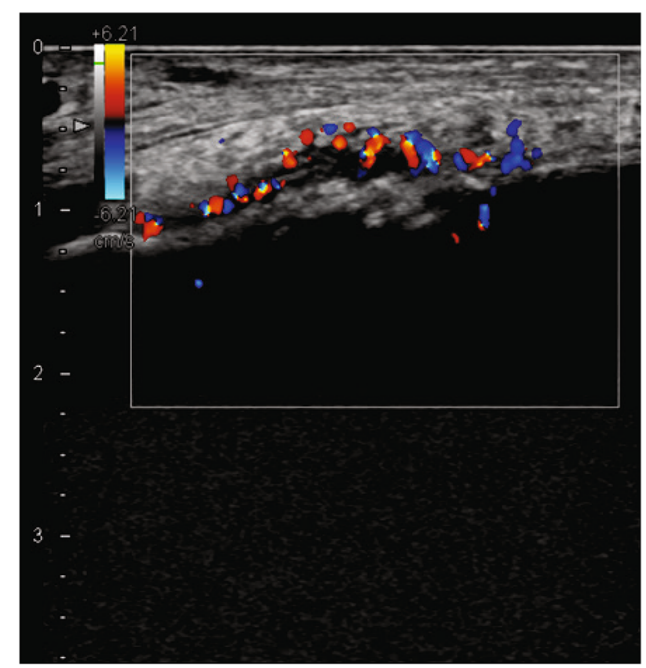

a

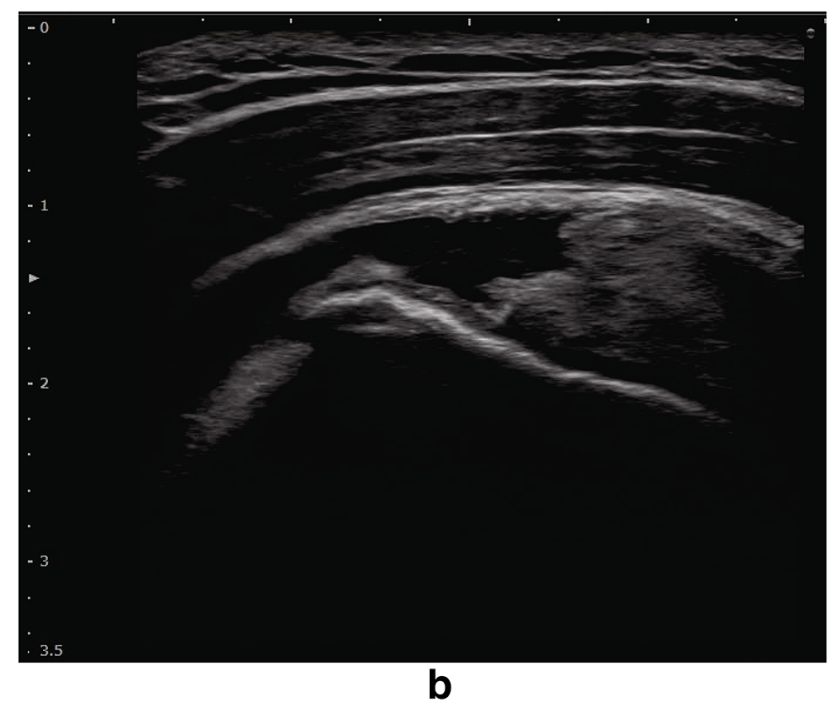

b

Fig. 1 High-resolution MSK ultrasonography. a Third metatarsal bone stress fracture, b rotator cuff tear 

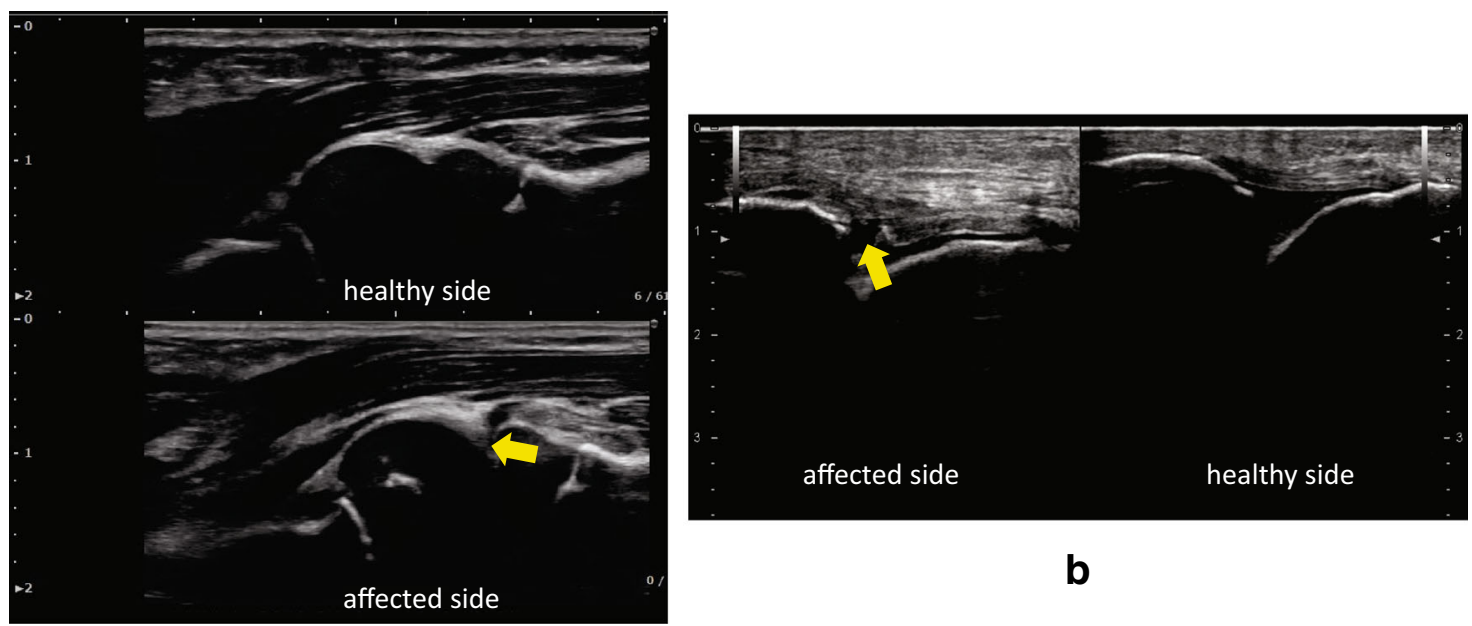

b

a

Fig. 2 High-resolution MSK ultrasonography. a Pulled elbow (arrow: interlocked annular ligament), b ATFL injury (arrow: partial tear)

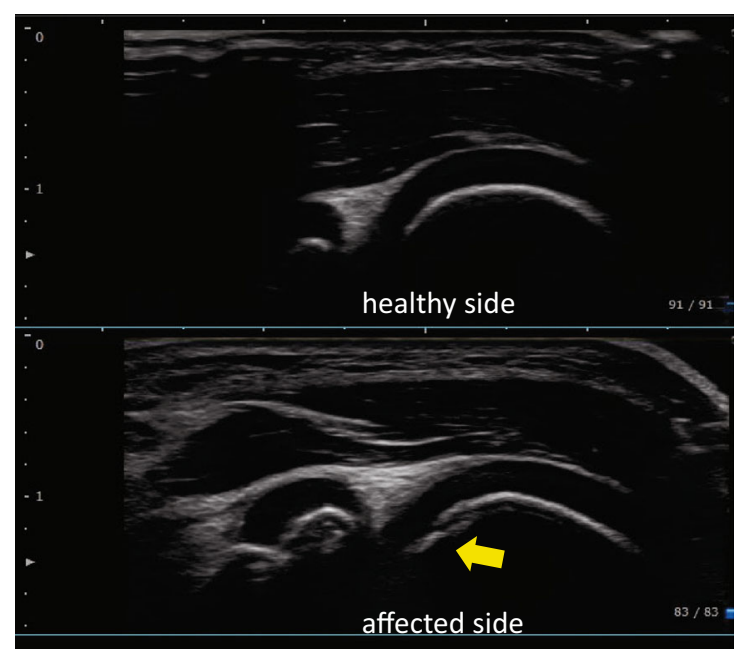

a

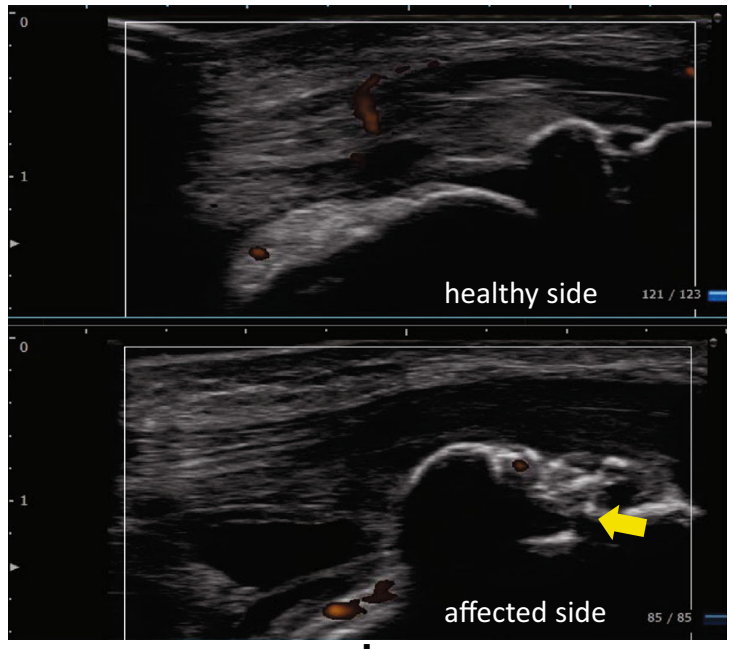

b

Fig. 3 High-resolution MSK ultrasonography. a Osteochondritis dissecans (arrow: capitulum is now irregular), b Osgood-Schlatter disease (arrow: the tibial tuberosity which appears as bony irregularity)

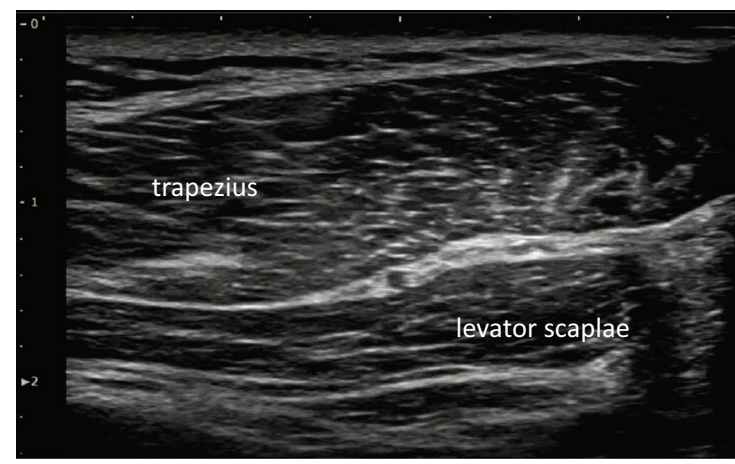

a

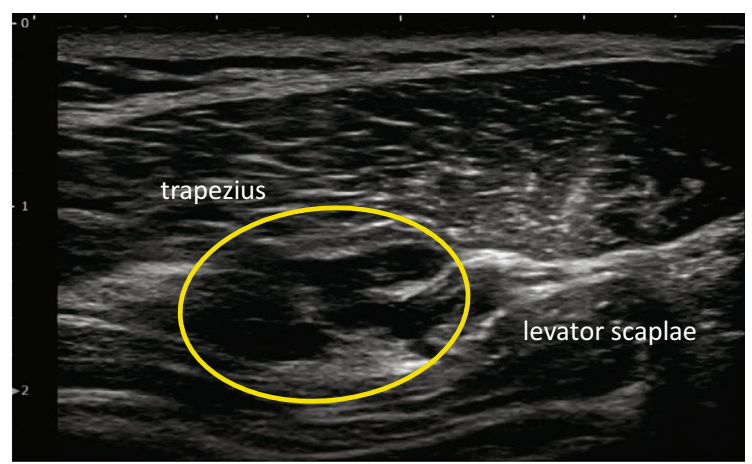

b

Fig. 4 US-guided hydro-release. a Pre-injection, $\mathbf{b}$ after injection (circle area: salin) 
making it possible to instantly alleviate pain using low-cost agents.

\section{Future of musculoskeletal ultrasound}

Musculoskeletal ultrasound is already being used in clinical settings as a tool for accurate diagnosis followed by immediate precise treatment. In the future, musculoskeletal ultrasound will further evolve into a tool for minimally invasive surgery. The current advances in musculoskeletal ultrasound will bring forth a new era of ultrasound-guided surgery. Arthroscopic surgery may someday be superseded by ultrasound-guided surgery, musculoskeletal ultrasound will make establishing more minimally invasive therapeutic procedures possible. This in turn will enable patients to return their normal lives sooner. Musculoskeletal ultrasound as an entity has evolved far quicker in a quarter of the time it took ultrasound itself to evolve in the last 60 years. Musculoskeletal ultrasound will and must continue to evolve in the future. Musculoskeletal ultrasound must continue to evolve for one simple reason and to borrow from what Steve Jobs had once said, "We can't just ask patients what they want and then try to give that to them. By the time we get it built, they'll want something new". 